\title{
Vibration reduction of a cantilever beam subjected to parametric excitation using time delay feedback
}

\author{
Yasser A. Amer ${ }^{1}$, and Sabry M. Elshourbagy ${ }^{2}$ \\ ${ }^{1}$ Mathematics Department,Faculty of Science, Zagazig University, Egypt. \\ 1 yaser31270@yahoo.com \\ 2 Department of Basic Sciences, Higher Technological Institute. 10 ${ }^{\text {th }}$ Ramadan City, Egypt. \\ 2 sabry.mohamed1315@yahoo.com \\ yaser31270@yahoo.com
}

\begin{abstract}
In this paper, dynamical behavior of a cantilever beam subject to parametric excitation under state feedback control with time delay is analyzed. The method of multiple scale perturbation technique is applied to obtain the solution up to the first order approximation. We obtain equations for the amplitude and phase. We studied all resonance cases numerically. Stability of the steady state solution for the selected resonance case is studied applying Rung-Kutta fourth method and frequency response equation via Matlab 7.0 and maple 16. From the results, it can be seen that the frequency and amplitude responses for the selected resonance case can be affected by the time delayed control. Effects of different parameters of the system are studied.
\end{abstract}

Keywords: Cantilever Beam; Time Delay; Vibration Control; Resonance Cases; Parametric Excitation.

\section{INTRODUCTION}

Over the last few years, numerous papers have been dedicated to the control of resonantly forced systems in various engineering fields. In passive vibration absorbers, a physical device is connected to the primary structure, while in the case of active absorbers, the device is replaced by a control system of sensors, actuators and filters. Active control of mechanical and structural vibrations is superior to passive control, because the former is more flexible in many aspects. Nayfeh and Nayfeh [1] utilized time-delay acceleration feedback to enhance stability for controlling machine-tool chatter. El-Bassiouny [2-3] presented the analysis of primary and sub harmonic resonances of a cantilever beam under time-delay feedback control using averaging and multiple scales methods. A number of important analytical conclusions were reached on the effect of feedback gains, the time-delay, the coefficient of cubic terms, and external excitations [4]. Qaroush and Daqaq [5] utilized a delayed feedback controller to reduce the vibrations of a macro cantilever beam and a micro cantilever sensor.

Erneux [6] investigated strongly nonlinear crane oscillations and lasers subject to optoelectronic feedback by the method of averaging, considering weak damping and weak feedback. Rand et al. [7] utilized a two-variable expansion perturbation scheme to analyze Van der Pol-Hopf bifurcation with delayed feedback. Naik and Singru [8] studied the resonance, stability and chaotic vibration of a quarter-car vehicle model with time-delay feedback. Xu et al. [9] investigated the effect of a time delay in a structure consisting of a linear beam and the NSC absorber. The analytical results based on the multiple scales method revealed much more complex dynamics for the system. The presence of the time delay widened or reduced the frequency bandwidth of effective vibration reduction.

Lei et al. [10] applied an active control technique to coordinate a kind of two parametrically excited chaotic system. Shen et al. [11] used incremental harmonic balance method to analyze nonlinear dynamics of a spur gear system including time varying stiffness and backlash. O'Malley and Kirkinis [12] with the aid of examples, illustrated the use of the RGM for singularly perturbeddifferential equations. They [13] used a combined RGM-multiple scale method forsingularly perturbed problems. Yingli et al. [14] studied Dynamic effects of delayed feedback control on nonlinear vibration floating raft systems. Gao, and Chen [15] studied Nonlinear analysis, design and vibration isolation for a bilinear system with time-delayed cubic velocity feedback.

The objective of this work is to study dynamical behavior of a cantilever beam subject to parametric excitation under state feedback control with time delay. The method of multiple scale perturbation technique is applied to obtain the solutionup to the second order approximation.

\section{MATHEMATICAL MODELING:}

This work is concerned with dynamical behavior of a cantilever beam subject to parametric excitation under state feedback control with time delay whose response is governed by a non linear partial differential equation

$$
\ddot{X}+\varepsilon \mu \dot{X}+X+\varepsilon \alpha X^{3}+\varepsilon \beta \dot{X} X^{2}+\varepsilon \gamma \ddot{X} X^{2}=-\varepsilon v \dot{X}(t-\tau)+X \varepsilon f \cos (\phi t)
$$

where a dot denotes differentiation with respect to time, $\mu$ the damping coefficient, $\alpha$ the curvature nonlinearity coefficients, $\beta$ and $Y$ are the inertia nonlinearity coefficients, $f$ is the amplitude of the parametric excitation, $\phi$ is the corresponding frequency, $v$ is the feedback gains and $\tau$ is the time delay. 


\subsection{MATHEMATICAL TECHNIQUES:}

Multiple scale perturbation method is conducted to obtain the approximate solution of Eq. (1). Assuming the solution in the form:

$$
\mathrm{X}(t ; \varepsilon)=X_{0}\left(T_{0}, T_{1}\right)+\varepsilon X_{1}\left(T_{0}, T_{1}\right)+\varepsilon^{2} X_{2}+O\left(\varepsilon^{3}\right)
$$

where $T_{0}=t$ and $T_{n}=\varepsilon^{n} t,(\mathrm{n}=0,1)$ are the fast and slow time scales, respectively. In terms of $T_{0}, T_{1}$ the times derivatives transform are:

$$
\begin{aligned}
& \frac{d}{d t}=D_{0}+\varepsilon D_{1}, \frac{d^{2}}{d t^{2}}=D_{0}^{2}+2 \varepsilon D_{0} D_{1}+\varepsilon^{2} D_{1}^{2} \\
& \text { where } D_{n}=\frac{\partial}{\partial T_{n}},(\mathrm{n}=0,1) .
\end{aligned}
$$

Substituting Eqs. (2) and (3) into Eq. (1) and equating the coefficients of the same power of $\varepsilon$ in both sides, we obtain:

$$
\begin{aligned}
& O\left(\varepsilon^{0}\right) \\
& \left(D_{0}^{2}+1\right) x_{0}=0 \\
& O(\varepsilon): \\
& \left(D_{0}^{2}+1\right) x_{1}=-2 D_{0} D_{1} x_{0}-\mu D_{0} x_{0}-\alpha x_{0}^{3}-\beta x_{0}^{2} D_{0} x_{0}-\gamma x_{0}^{2} D_{0}^{2} x_{0}-v D_{0} x_{0 \tau}+f x_{0} \cos (\phi t) \\
& O\left(\varepsilon^{2}\right) \quad-\gamma x_{0}^{2} D_{0}^{2} x_{1}-2 \gamma x_{0}^{2} D_{0} D_{1} x_{0}-2 \gamma x_{0} x_{1} D_{0}^{2} x_{0}-v D_{1} x_{0 \tau}+f x_{1} \cos (\phi t) \\
& \left(D_{0}^{2}+1\right) x_{2}=-2 D_{0} D_{1} x_{1}-D_{1}^{2} x_{0}-\mu D_{0} x_{1}-\mu D_{1} x_{0}-3 \alpha x_{0}^{2} x_{1}-\beta x_{0}^{2} D_{0} x_{1}-\beta x_{0}^{2} D_{1} x_{0}-2 \beta x_{0} x_{1} D_{0} x_{0}
\end{aligned}
$$

The general solution of Eq. (4) is

$$
\begin{aligned}
& x_{0}\left(T_{0}, T_{1}\right)=A\left(T_{1}\right) e^{i T_{0}}+\bar{A}\left(T_{1}\right) e^{-i T_{0}} \\
& x_{0 \tau}\left(T_{0}\right)=A\left(T_{1}\right) e^{i\left(T_{0}-\tau\right)}+\bar{A}\left(T_{1}\right) e^{-i\left(T_{0}-\tau\right)}
\end{aligned}
$$

where $A$ is a complex function in $T_{1}$, which can be determined from eliminating the secular terms at the next approximation. Substituting Eq. (7-8) into Eq. (5), then we have that:

$$
\begin{aligned}
\left(D_{0}^{2}+1\right) x_{1}= & \left(-2 i D_{1} A-i \mu A-3 \alpha A^{2} \bar{A}-i \beta A^{2} \bar{A}+3 \gamma A^{2} \bar{A}-i v A e^{-i \tau}\right) e^{i T_{0}}+\left(-\alpha A^{3}-i \beta A^{3}+\gamma A^{3}\right) e^{3 i T_{0}} \\
& +\frac{f}{2}\left(A e^{i(1+\phi) T_{0}}+\bar{A} e^{i(-1+\phi) T_{0}}\right)+\mathrm{cc}
\end{aligned}
$$

where cc denotes the complex conjugate, after elimination the secular terms then the solution of Eq. (9) can be written as:

$$
x_{1}\left(T_{0}, T_{1}\right)=E_{1} e^{3 i T_{0}}+E_{2} e^{i(1+\phi) T_{0}}+E_{3} e^{i(-1+\phi) T_{0}}+c c
$$

Substituting Eq. (7-8) and (10) into Eq. (6), then we have that: 


$$
\begin{aligned}
\left(D_{0}^{2}+1\right) x_{2}= & {\left[-D_{1}^{2} A-2 i D_{2} A-\mu D_{1} A-3 \alpha E_{1} \bar{A}^{2} A^{3}-i \beta E_{1} \bar{A}^{2} A^{3}-\beta\left(D_{1} A\right) A^{2}-2 \beta\left(D_{1} A\right) A \bar{A}+11 \gamma E_{1} A^{3} \bar{A}\right.} \\
& \left.-4 i \gamma\left(D_{1} A\right) A \bar{A}+2 i \gamma\left(D_{1} A\right) A^{2}-v\left(D_{1} A\right) e^{-i \tau}-\frac{1}{2} E_{2} f A\right] e^{i T_{0}}+\left[-6 i E_{1} A^{3}-3 i E_{1} \mu A^{3}-6 E_{1} \alpha A^{4} \bar{A}\right. \\
& \left.-6 i E_{1} A^{4} \bar{A}+22 E_{1} \gamma A^{4} \bar{A}-2 i \gamma\left(D_{1} A\right) A^{2}-\beta\left(D_{1} A\right) A^{2}-3 i E_{1} v A^{3}\right] e^{3 i T_{0}}+\left[-3 E_{1} \alpha A^{5}-5 i E_{1} \beta A^{5}\right. \\
& \left.+11 E_{1} \gamma A^{5}\right] e^{5 i T_{0}}+\left[-2 i E_{2}(1+\phi) A-i E_{2} \mu(1+\phi) A+6 E_{2} \alpha A^{2} \bar{A}+4 i E_{1} \beta(1+\phi) A^{2} \bar{A}\right. \\
& \left.-2 E_{2} \gamma(1+\phi)^{2} A^{2} \bar{A}-2 E_{2} \gamma A^{2} \bar{A}+i E_{2} v(1+\phi) e^{-i(1+\phi) \tau}\right] e^{i(1+\phi) T_{0}}+\left[3 E_{2} \alpha \bar{A}^{2} A-2 i E_{2} \beta(1+\phi) \bar{A}^{2} A\right. \\
& \left.-2 i E_{2} \beta \bar{A}^{2} A-E_{2} \gamma(1+\phi)^{2} \bar{A}^{2} A-2 E_{2} \gamma \bar{A}^{2} A\right] e^{i(-1+\phi) T_{0}}+\frac{1}{2} E_{1} f A^{3} e^{i(3-\phi) T_{0}}+\left[3 E_{2} \alpha A^{3}\right. \\
& \left.+i E_{2} \beta(1+\phi) A^{3}+2 i E_{2} A^{3}-E_{2} \gamma(1+\phi)^{2} A^{3}-2 E_{2} \gamma A^{3}+\frac{1}{2} E_{1} f A^{3}\right] e^{i(3+\phi) T_{0}} \\
& -\frac{1}{2} E_{2} f A e^{i(1+2 \phi) T_{0}}
\end{aligned}
$$

Elimination the secular terms then the solution of Eq. (11) can be written as:

$$
x_{2}\left(T_{0}, T_{1}\right)=E_{4} e^{3 i T_{0}}+E_{5} e^{5 i T_{0}}+E_{6} e^{i(1+\phi) T_{0}}+E_{7} e^{i(-1+\phi) T_{0}}+E_{8} e^{i(3-\phi) T_{0}}+E_{9} e^{i(3+\phi) T_{0}}+E_{10} e^{i(1+2 \phi) T_{0}}+c c
$$

where $E_{j},(j=1 \ldots 3)$ are complex functions of $T_{1}$.

\section{STABILITY ANALYSIS:}

We study the different resonance cases numerically to see the worst case, one of the worst cases has been chosen to study the system stability. The selected resonance case $\phi=2$. In this case we introduce the detuning parameter $\sigma$ according to

$$
\phi=2+\varepsilon \sigma
$$

Substituting Eq. (13) into Eq. (9), then eliminating the secular and small divisor terms from $x_{1}$, we get the following :

$$
-2 i D_{1} A-i \mu A-3 \alpha A^{2} \bar{A}-i \beta A^{2} \bar{A}+3 \gamma A^{2} \bar{A}-i v A e^{-i \tau}+\frac{f}{2} \bar{A} e^{i \sigma T_{1}}=0
$$

To analyze the solution of equation (14) it is convenient to express $A$ in the polar form:

$$
A\left(T_{1}\right)=\frac{1}{2} a\left(T_{1}\right) e^{i \theta\left(T_{1}\right)}
$$

wherea and $\boldsymbol{\theta}$ and are unknown real-valued functions. Inserting Eq. (15) into Eq. (14) and separating real and imaginary parts, we have

$$
\begin{aligned}
& a \theta^{\prime}=\frac{3}{8}(\alpha-\gamma) a^{3}+\frac{v}{2} a \sin \tau-\frac{f}{4} a \cos \varphi \\
& a^{\prime}=-\frac{\mu}{2} a-\frac{\beta}{8} a^{3}-\frac{v}{2} a \cos \tau+\frac{f}{4} a \sin \varphi
\end{aligned}
$$

where $\varphi=\sigma T_{1}-2 \theta$

For steady state solutions $a^{\prime}=\varphi^{\prime}=0, \theta^{\prime}=\frac{1}{2} \sigma$ obtained

$$
\begin{aligned}
& f \cos \varphi=\frac{3}{2}(\alpha-\gamma) a^{2}+2 v \sin \tau-2 \sigma \\
& f \sin \varphi=2 \mu+\frac{a^{2}}{2} \beta+2 v \cos \tau
\end{aligned}
$$

From equations (18-19) the frequency response equation is given by:

$$
\left(\frac{3}{2}(\alpha-\gamma) a^{2}+2 v \sin \tau-2 \sigma\right)^{2}+\left(2 \mu+\frac{\beta}{2} a^{2}+2 v \cos \tau\right)^{2}-f^{2}=0
$$




\subsection{LINEAR SOLUTIONS:}

Now, to study the stability of the linear solution of the obtained fixed let us consider $A$ in the form:

$$
A\left(T_{1}\right)=\frac{1}{2}(p-i q) e^{i \delta T_{1}}
$$

where $p$ and $q$ are real values functions in $T_{1}$ and consider $2 \delta=\sigma$.

Substituting from Eq. (21) into the linear parts of Eq. (14) and separating real and imaginary parts, the following system of equations are obtained:

$$
\begin{aligned}
& p^{\prime}+\left(\frac{\mu}{2}+\frac{v}{2} \sin \tau\right) p+\left(\delta+\frac{v}{2} \cos \tau-\frac{f}{4}\right) q=0 \\
& q^{\prime}+\left(-\delta-\frac{v}{2} \cos \tau-\frac{f}{4}\right) p+\left(\frac{\mu}{2}+\frac{v}{2} \sin \tau\right) q=0 \\
& \left|\begin{array}{cc}
\frac{1}{2}(\mu+v \sin \tau)-\lambda & -\left(\delta+\frac{v}{2} \cos \tau-\frac{f}{4}\right) \\
\left(\delta+\frac{v}{2} \cos \tau+\frac{f}{4}\right) & -\frac{1}{2}(\mu+v \sin \tau)-\lambda
\end{array}\right|=0
\end{aligned}
$$

where,

$$
\lambda_{1,2}=-\frac{v}{2} \pm \frac{1}{4} \sqrt{4 v^{2} \sin ^{2}(\tau)+8 \mu \sin (\tau)-16 \mu+16 \delta v \cos (\tau)+4 \mu^{2}+4 v^{2}+16 \delta^{2}-f^{2}}
$$

The linear solution is stable in this case if and only if the real parts of $v>0$, and otherwise it is unstable.

\subsection{NON- LINEAR SOLUTIONS:}

To determine the stability of the fixed points, one lets

$$
a=a_{10}+a_{11} \text { and } \varphi=\varphi_{10}+\varphi_{11}
$$

where $a_{10}, \varphi_{10}$ are solutions of Eqs. (18), (19) and $a_{11}, \varphi_{11}$ are perturbations which are assumed to be small comparing to $a_{10}, \varphi_{10}$.substituting Eq.(25) into Eqs.(16) and (17) using Eqs. (18) and (19) and keeping only the linear terms in $a_{11}$, $\varphi_{11}$ we obtain:

$$
\begin{aligned}
& a_{11}^{\prime}=\left(\frac{-\mu}{2}-\frac{3 \beta}{8} a_{10}^{2}+\frac{v}{2} \cos \tau+\frac{f}{4} \sin \varphi_{10}\right) a_{11}+\left(\frac{f}{4} a_{10} \cos \varphi_{10}+\frac{f}{4} a_{11} \cos \varphi_{10}\right) \varphi_{11} \\
& \varphi_{11}^{\prime}=\left(\frac{\sigma}{a_{10}}-\frac{9}{8}(\alpha-\gamma) a_{10}+\frac{v}{2 a_{10}} \sin \tau+\frac{f}{4 a_{10}} \cos \varphi_{10}\right) a_{11}-\left(\frac{f}{4} \sin \varphi_{10}+\frac{f}{4 a_{10}} a_{11} \sin \varphi_{10}\right) \varphi_{11} \\
& \left|\begin{array}{ll}
\frac{-\mu}{2}-\frac{3 \beta}{8} a_{10}^{2}+\frac{v}{2} \cos \tau+\frac{f}{4} \sin \varphi_{10} & \frac{f}{4} a_{10} \cos \varphi_{10} \\
\frac{\sigma}{a_{10}}-\frac{9}{8}(\alpha-\gamma) a_{10}+\frac{v}{2 a_{10}} \sin \tau+\frac{f}{4 a_{10}} \cos \varphi_{10} & -\left(\frac{f}{4} \sin \varphi_{10}\right)
\end{array}\right|=0
\end{aligned}
$$

Consequently, a non-linear solution is stable if and only if the real parts of both eigen values of the coefficient matrix (28) are less than zero.

\section{NUMERICAL RESULTS:}

The differential equation of the system(1) is solved numerically using fourth order RungeKutta method at non resonance case without control as shown in Fig. 1 from this figure the system is stable with amplitude about 0.15 which considered as basic case. From Fig.2 we can see that the system at resonance case $\phi=2$ without control the amplitude is increased to about 0.85 (six times) compared with the basic case in Fig.1. The effect of delay time on system response is shown in Fig. 3 the amplitude is reduced to minimum valueat $\tau=0.1$, which mean that the control is effective. Figure 4 show the effect of time delay on the main system we can observe that for $0.1 \leq \tau \leq 1.5,5.1 \leq \tau \leq 7.6,11.6 \leq \tau \leq 13.6$ and $18.1 \leq \tau \leq 19.6$ the amplitude is reduced to minimum value otherwise the system amplitude is increase. 

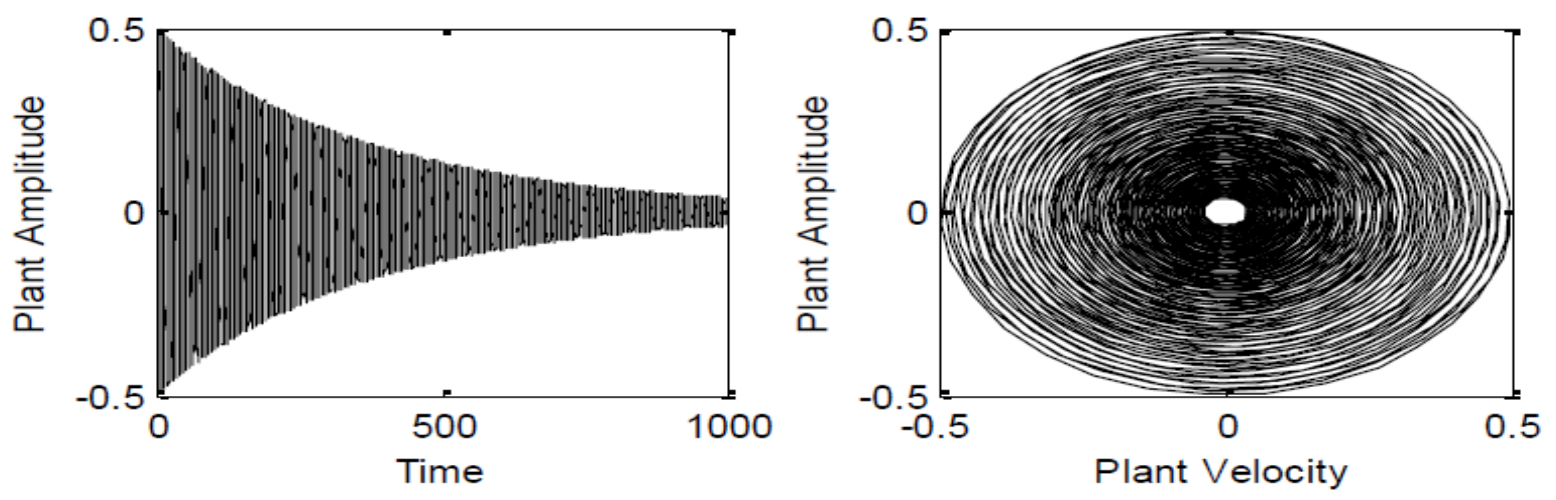

Fig.1: System behavior without control in basic case
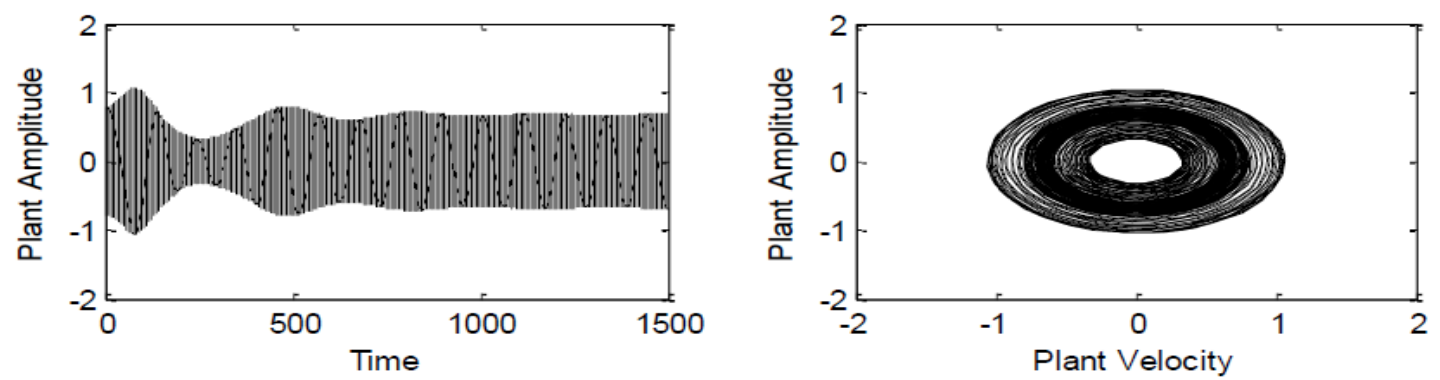

Fig.2: System behavior without control in resonance case $\phi=2$
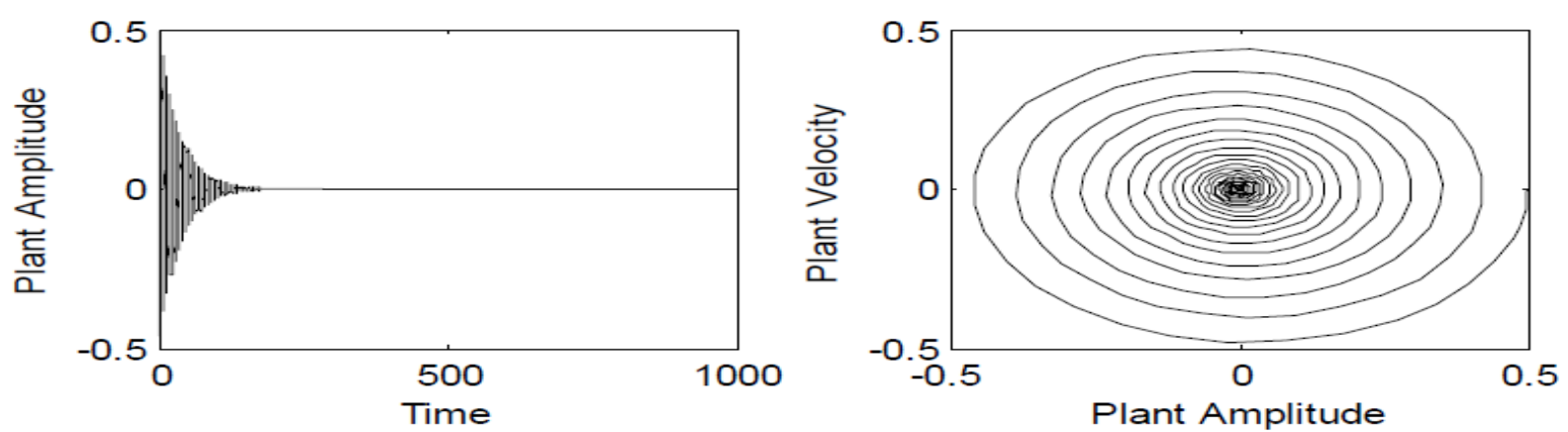

Fig.3: System behavior with time delayed at resonance case $\phi=2$

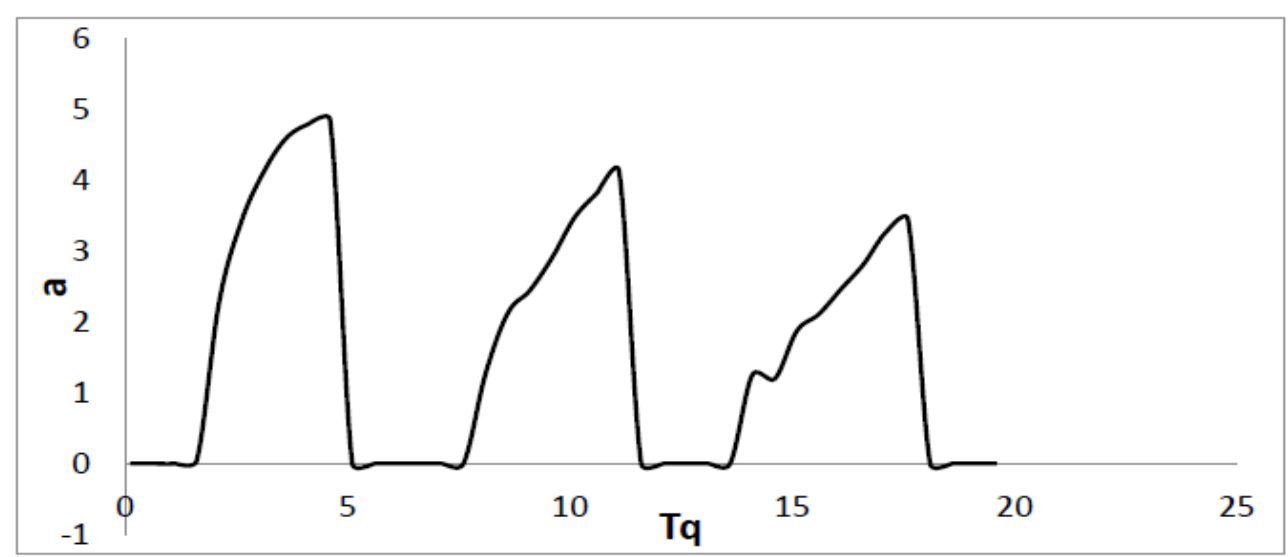

Fig.4: The effect of delay

\subsection{FREQUENCY RESPONSE CURVES:}

The frequency response equation (20) is a non-linear algebraic equation in the amplitude $a$. This equation is solved numerically and the results are shown in the fig.5, which representing the vibration of the amplitude $a$ against the detuning parameter $\sigma$ for the given values of the other parameters. The steady state amplitude is monotonic deceasing function of 
the gain $V$, the linear damping $\mu$, the curvature nonlinearity coefficients $\alpha$, the inertia nonlinearity coefficients $\beta$ and $\gamma$ as shown in Figure $5 \mathrm{a}, 5 \mathrm{~d}, 5 \mathrm{e}$ and $5 \mathrm{f}$ respectively. But the state amplitude is monotonic increasing function of the excitation force amplitude $f$ as shown in Figure $5 \mathrm{c}$
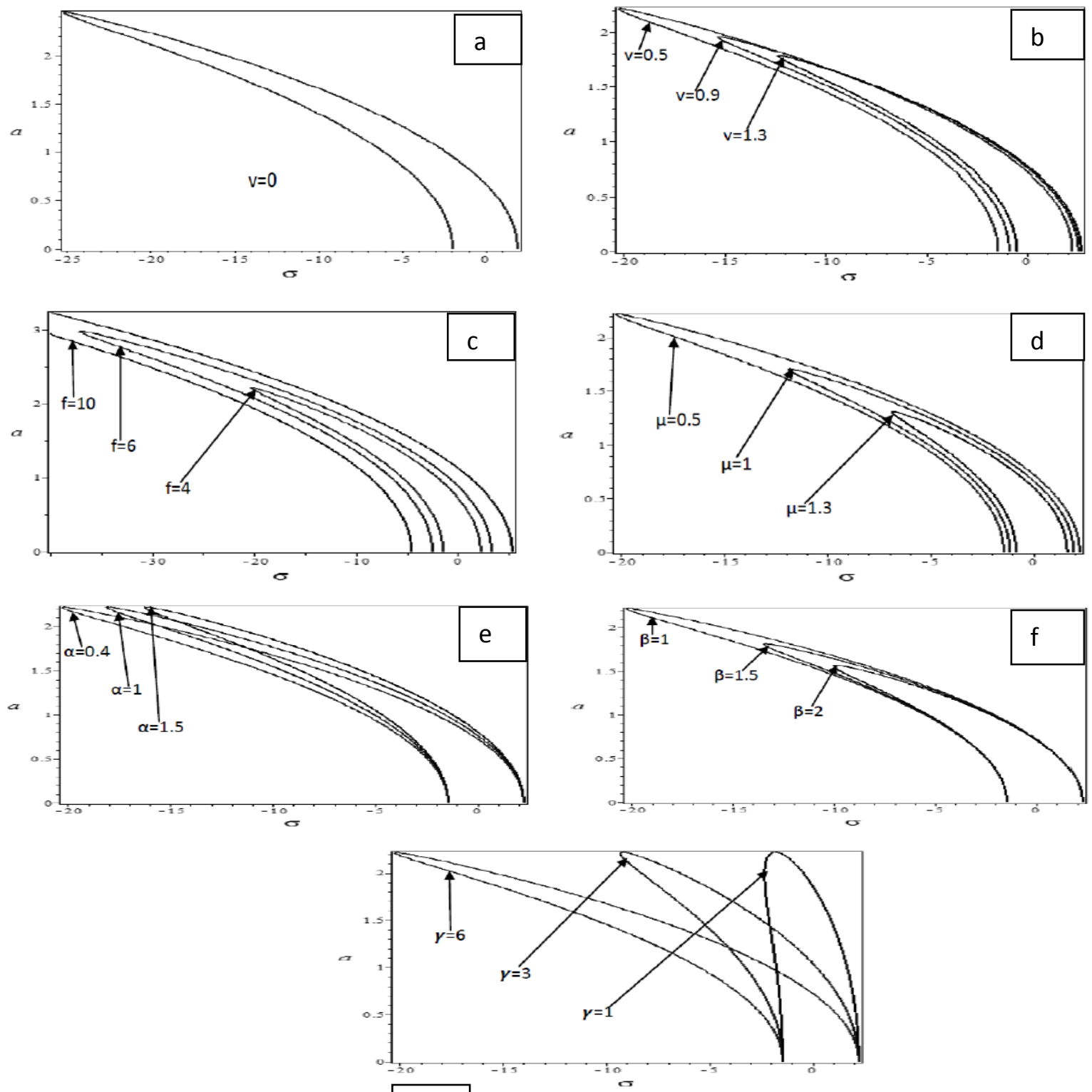

Fig.5: Frequen $g$ onse curve for $f=4, \alpha=0.4, \beta=1, y=6, \mu=0.5, v=0.5$

\subsection{EXCITATION RESPONSE CURVES:}

Fig. 6, shows the force response equation (20) is a nonlinear algebraic equation of the amplitude a, which is solved numerically. This equation representing the vibration of the amplitude a against the excitation force amplitude $f$, for the given values of the other parameters. The steady state amplitude is monotonic increasing function of the gain $V$ and the curvature nonlinearity coefficients $\alpha$ as shown in Figure $6 \mathrm{~b}$ and $6 \mathrm{~d}$. But the state amplitude is monotonic deceasing function of the linear damping $\mu$ as shown in Figure 6c.
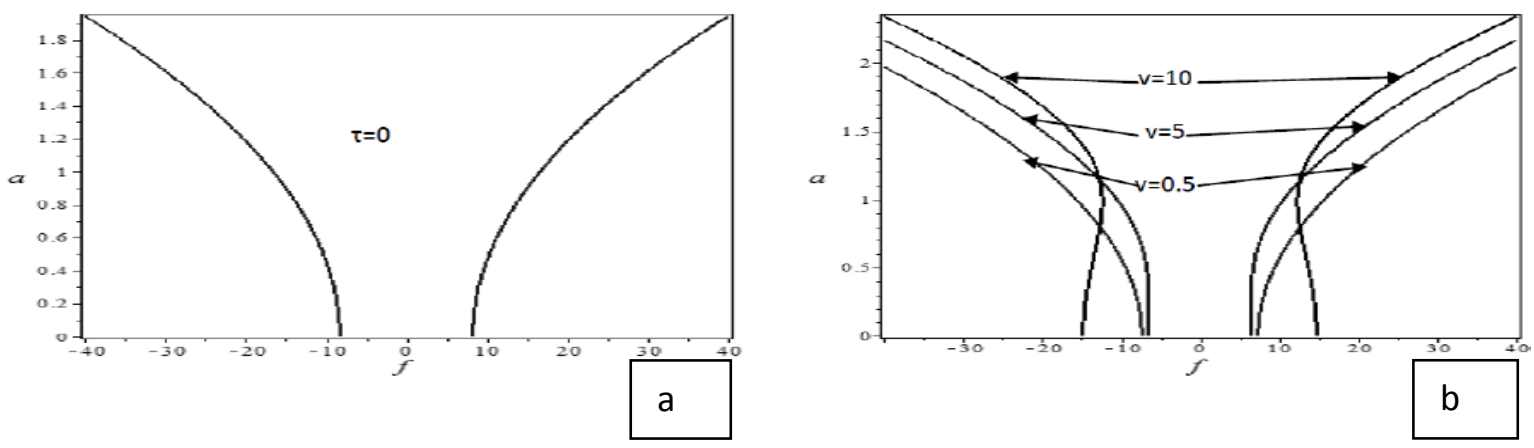


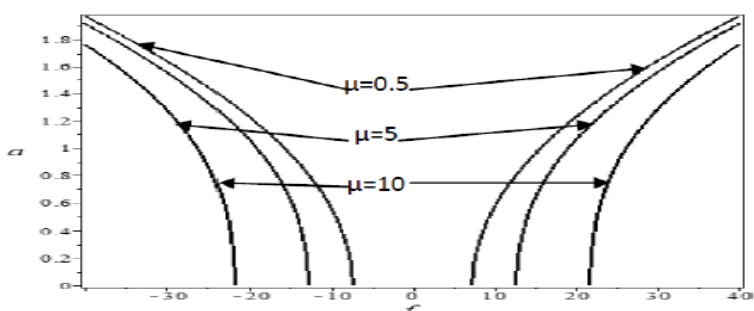

Fig.6: Frequency excitation curves (The vibration $\mathrm{C}$ $\beta=1, y=6, \mu=0.5, v=0.5$ )

\section{Comparison between numerical solution and approximate solution}

Now, we get a good agreement of the approximate solution obtained from frequency response equation and thenumerical solution obtained by Runge-Kutta-fourth-order method as shown in Figure 7.

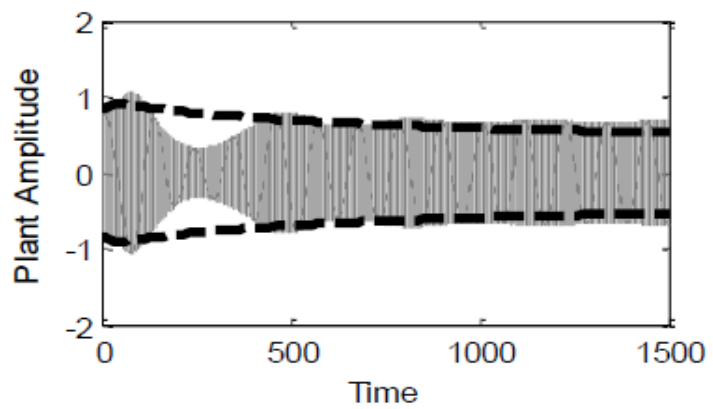

Fig.7: Comparison between the numerical solution

\section{CONCLUSIONS:}

The dynamical behavior of a cantilever beam subject to parametric excitation is investigated. The method ofmultiple scale perturbation technique is applied to obtain the solution up to the second order approximation. At first westudied the system behavior without control and the effect of parameters at this case. The time delayed control of negativefeedback with linear velocity is used to vibration control. All resonance cases are studied numerically to obtainthe worst case. Stability of the steady state solution for the selected resonance case is studied applying Rung-Kutta fourth method and frequency response equation. From the above study the following may be concluded:

1- The worst resonance case is the sub harmonic resonance $\phi \cong 2$, which leads to the amplitudes of the main systemare increased to about 0.85 ( 6 times) of the basic case.

2-The time delayed controls of negative feedback with linear velocity is effective and reduce the amplitude of vibration.

3-The steady state amplitude is monotonic deceasing function of the gain $v$, the linear damping $\mu$, the curvature nonlinearity coefficients $\alpha$, the inertianonlinearity coefficients $\beta$ and $\gamma$. But the state amplitude is monotonic increasing function of the excitation forceamplitude $f$.

4- There is a good agreement between the numerical solution and the approximate solution.

\section{REFERENCES:}

1. Nayfeh, A., Nayfeh, H.,N. A. 2012. Time-delay feedback control of lathe cutting tools. J. Vib. Control 18, 11061115.

2. El-Bassiouny, A. F. 2006. Fundamental and sub-harmonic resonances of harmonically oscillation with time delay state feedback. Shock Vib. 13, 65-83.

3. El-Bassiouny, A.F. 2006. Vibration control of a cantilever beam with time delay state feedback. Z. Naturforsch. A, J. Phys. Sci. 61, 629-640.

4. El-Bassiouny, A.F., El-Kholy, S. 2010. Resonances of a nonlinear single-degree-of-freedom system with time delay in linear feedback control. Z. Naturforsch. A, J. Phys. Sci. 65, 357-368.

5. Qaroush, Y., Daqaq, M. F. 2010. Vibration mitigation in multi-degree-of-freedom structural systems using filteraugmented delayed-feedback algorithms. Smart Mater. Struct. 19, 085016. 
6. Erneux, T. 2010. Strongly nonlinear oscillators subject to delay. J. Vib. Control 16, 1141-1149.

7. Rand, R. H. Suchorsky, M. K., Sah, S. M. 2010. Using delay to quench undesirable vibrations. Nonlinear Dyn. 62 , 407-416.

8. Raghavendra D. Naik, Pravin M. Singru 2011. Resonance stability and chaotic vibration of a quarter-car vehicle model with time-delay feedback, Commun.Nonlinear Sci. Numer. Simulat. 16, 3397-3410.

9. Xu, J., Chung, K.W. and Zhao, Y.Y. 2010. Delayed saturation controller for vibration suppression in a stainlesssteel beam, Nonlinear Dynamic 62, 177-193.

10. Lei, Y., Xu, W., Shen, J. Fang, T. 2006. Global synchronization of two parametrically excited systems using active control. Chaos Solitons Fractals 28, 428-436.

11. Shen, Y., Yang, S., Liu, X. 2006. Nonlinear dynamics of a spur gear pair with time-varying stiffness and backlash based on incremental harmonic balance method. Journal of Mechanical Sciences 48, 1256-1263.

12. O'Malley, R.E., Kirkinis, E. 2009. Examples illustrating the use of renormalization techniques for singularly perturbed differential equations, Stud. Appl. Math. 122 (2) 105-122.

13. O'Malley, R.E. Kirkinis, E. 2010. A combined renormalization group-multiple scale method for singularly perturbed problems, Stud. Appl. Math. 124, 383-410.

14. Yingli Li, Daolin Xu, Yiming Fu, and Jing Zhang 2014. Dynamic effects of delayedfeedback control on nonlinear vibration floating raft systems, Journal of Sound andVibration, 33, 2665-2676.

15. Gao, X., and Chen, Q. 2014. Nonlinear analysis, design and vibration isolation for abilinear system with timedelayed cubic velocity feedback, Journal of Sound andVibration, 33, 1562-1576

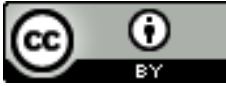

This work is licensed under a Creative Commons Attribution 4.0 International License. 